\title{
A Generalized Power-Based Modelica Library with Application to an Industrial Hydraulic Plant
}

\author{
Ilja Alkov, Robin Diekmann, Dirk Weidemann \\ Institute of System Dynamics and Mechatronics \\ University of Applied Sciences Bielefeld \\ Wilhelm-Bertelsmann-Str. 10, 33602 Bielefeld, Germany \\ \{ilja.alkov, robin.diekmann, dirk.weidemann\}@fh-bielefeld.de
}

\begin{abstract}
This contribution presents a Modelica implementation of the generalized power-based modeling approach conforming to the bond graph methodology. The corresponding developed library BondGraph is discussed in detail. It allows graphical modeling according to the bond graph formalism, and contains common bond graph elements, as well as specific nonlinear elements, especially related to hydraulic effects. Furthermore, several composed models are provided, such as switching valves, pipes, cylinders, etc. A combination with blocks of the Modelica Standard Library is possible. The application of BondGraph to an industrial plant is described to demonstrate its capabilities.
\end{abstract}

Keywords: power-based model, bond graph, library, Modelica, hydraulic

\section{Introduction}

Since general purpose simulation environments enhance their capabilities, modelers attempt to take advantage of the offered possibilities completely. Hence, models become more comprehensive and cover the multidisciplinarity of the considered systems. Therefore, domain specific modeling libraries are developed and offer possibilities to model the involved different physical effects separately as well as to interconnect them. The generalized power-based approach is an alternative efficient modeling formalism. Based on the generalized power definition, unified modeling elements are conceptualized applicable to the direct multidisciplinary modeling of complex systems. Consequently, the modeling procedure consists of the characterization of domain specific processes corresponding to their unified complements and interconnecting these complements to the model of complete sys- tem according to its structure. For further advantages of generalized power-based modeling, see e.g. [1] and [2]. The Modelica language is particularly suitable for the development and representation of powerbased models, primly because of the possibility of the acausal multiple signal connections definition.

The bond graph (BG) formalism provides a multidisciplinary, generalized power-based approach to the modeling and also graphical model representation of dynamic systems (cf. [3]). By the objectoriented nature, BG possess the according advantages. The graphical system representation by a BG can be translated automatically to a system of differentialalgebraic equations, wherefore the implementation of the BG approach with an appropriate modeling language is necessary.

The Modelica language and the BG formalism are partly closely related modeling methodologies. Hence, an implementation of the BG formalism in Modelica is considered in this contribution. The proposed implementation attempts to take advantages of both modeling concepts. The developed open source library BondGraph is recommended to be used with Dymola 7 or later versions as this software supports completely included model definitions.

The introduced BondGraph library is available online at the official Modelica web page [4]. It contains common standard BG elements, as well as specific nonlinear elements. The included nonlinear models were designed especially for the modeling of hydraulic networks. For these, attention has been paid to their numerically stable computation. Further components provided by BondGraph include composed models of technical units, e.g. valves, and blocks for signal generation, e.g. to control valves.

A short introduction to the BG formalism is given in the Section 2. The implementation of a number of representative elements in the BondGraph library is 
discussed in Section 3. In Section 3.1, the implementation of the standard BG elements, and in Section 3.2, the implementation of the hydraulic elements are presented. Section 4 introduces an industrial plant and the corresponding model developed using the BondGraph library. Several examples are provided to illustrate the described issues. A brief summary is given in Section 5. The used formula symbols are summarized in the Appendix.

\section{Bond Graphs}

Since its development, the BG approach has become a well-known technique for object-oriented graphical modeling (cf. [3]). This fact is based on the established definition of generalized power (cf. [5]) as the product of a conceptional effort $e$ and a flow $f$ variable:

$$
P=f \cdot e .
$$

This generalized specification provides the feasibility of multidisciplinary modeling with unified elements. The only prerequisite for this obviously advantageous modeling approach is an appropriate assignment of power variables i.e. effort and flow for considered domains.

For example, in the field of isothermal hydraulics, conventionally the pressure and the volumetric flow rate are assigned as the effort and the flow variable, respectively. A list of corresponding possible assignments for different energy domains is shown in Table 1.

Table 1: A selection of domains and corresponding conventional variable assignments in the bond graph formalism

\begin{tabular}{l|l|l}
\hline Domain & Flow & Effort \\
\hline hydraulic & volume flow rate & pressure \\
translational & velocity & force \\
rotational & angular velocity & torque \\
electrical & current & voltage \\
thermal & entropy flow rate & temperature \\
\hline
\end{tabular}

\subsection{Bond Graph Elements}

The basic set of BG elements consists of an effort source, a flow source, a capacitance, an inductance, a resistance, a transformer, and a gyrator, which are given with corresponding constitutive equations in Table 2.

Table 2: Basic bond graph elements

\begin{tabular}{l|c|l}
\hline Name & Element & Constitutive Equation \\
\hline effort source & $S e$ & $e=e_{s}$ \\
flow source & $S f$ & $f=f_{s}$ \\
capacitance & $C$ & $e=\frac{1}{c} \int_{t_{0}}^{t} f\left(t^{*}\right) d t^{*}+e\left(t_{0}\right)$ \\
inductance & $I$ & $f=\frac{1}{i} \int_{t_{0}}^{t} e\left(t^{*}\right) d t^{*}+f\left(t_{0}\right)$ \\
resistance & $R$ & $e=r \cdot f$ \\
transformer & $T F$ & $e_{1}=r_{t f} \cdot e_{2}, f_{1}=\frac{1}{r_{t f}} \cdot f_{2}$ \\
gyrator & $G Y$ & $e_{1}=r_{t f} \cdot f_{2}, f_{1}=\frac{1}{r_{t f}} \cdot e_{2}$ \\
\hline
\end{tabular}

A significant extension of the modeling opportunities is offered by modulated elements. These are generalizations of linear elements where the proportionality coefficients are given by an external signal.

Two further multi-port elements referred to as junctions are defined in the bond graph methodology. These may represent conservation or equilibrium laws but also design constraints among the variables of the elements connected. In the graphical representation, the two power variables are carried by one eponymous bond connecting junctions and other BG elements, that is usually drawn as a half arrow. The flows of all elements connected to a 1-Junction are equal, whereas the sum of efforts carried by all incoming bonds equals the sum of efforts carried by all outgoing bonds. Again a 0-Junction is a dual element corresponding to the 1Junction, hence for this the reversed relations of effort and flow are valid. For example, related to the hydraulic domain, all elements connected to a 1-Junction have the same flow as being connected in series, and all elements connected to a 0-Junction have the same effort as being connected in parallel.

Formally, a 1-Junction is described by the equations

$$
\begin{aligned}
& \sum_{k=1}^{n} s_{k} \cdot e_{k}=0, s_{k} \in\{+1,-1\}, \\
& f_{1}=f_{2}=\cdots=f_{n},
\end{aligned}
$$

where $n$ equals the number of elements connected to the junction. In the same manner, a 0-Junction is described by

$$
\begin{aligned}
& \sum_{k=1}^{n} s_{k} \cdot f_{k}=0, s_{k} \in\{+1,-1\}, \\
& e_{1}=e_{2}=\cdots=e_{n} .
\end{aligned}
$$


By convention, $s_{k}=+1$ for all incoming bonds and $s_{k}=-1$ for all outgoing bonds. Hence, the contributions to the constitutive equation of a junction are determined by the directions of the bonds in the graphical representation. Nevertheless, as can be seen by (2) and (4), formally, this is rather a property of the junction itself than of the connection. This fact is considered by the proposed implementation of the BG formalism in Modelica.

According to a specific domain, for each standard BG element a physical interpretation can be given. Considering a combination of linear elements, modulated elements, and sensors observing power variables, it is possible to describe a wide range of nonlinear models by case-related definitions of the external signal. Nevertheless, the definition of applicationspecific, and, where appropriate, nonlinear elements may lead to a convenient modeling process and ensures the clarity of the obtained models.

\subsection{Causality}

Within the BG formalism, bond connections are representations of information exchange between submodels. As two power variables are associated with each bond, two equations are obliged for their calculation and thus each end of the bond is responsible for one of them. Graphically, the flow determining end is marked by a short stroke. For example, energy storage elements are preferred to have integral causality, thus a capacitance preferably computes effort and an inductance preferably computes flow, whereas sources obviously determine their output variables. Several rules exist for causality assignment, e.g. listed in [3].

The graphical assignment of causality is useful if the equations describing the system behavior are directly deduced from the graphical BG representation. In some modeling tools as for example 20-sim (cf. [6]), the causality is fixed during the modeling procedure. However, it is not required to assign the causality during the modeling process using Modelica, as this is a subtask of the model compilation process, which is performed by suitable matching algorithms. For large models and generally for practical modeling, the manually or a priori fixed causality assignment is less reasonable. Therefore, manual causality assignment is not integrated in the presented BG implementation.

\subsection{Example}

An example of a simple physical system and its BG representation are shown in Fig. 1. The considered system is a hydraulic cylinder driven by enforced pressures at hydraulic ports assigned with $\mathrm{PT}_{1}$ and $\mathrm{PT}_{2}$. On the mechanical side, a load is executed represented by a force at the corresponding port $\mathrm{PT}_{3}$. In this simple representation, basic BG elements are utilized exclusively, which may be less suitable for modeling with practical accuracy requirements.

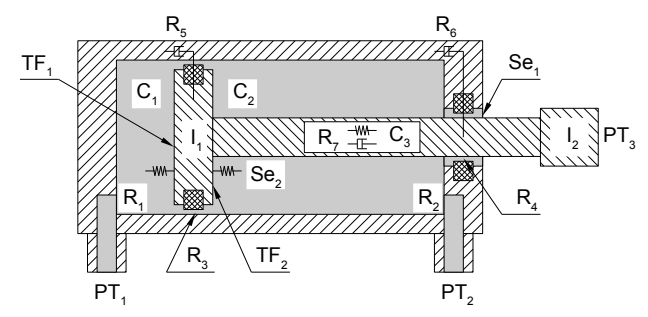

(a) Iconic model of a hydraulic cylinder

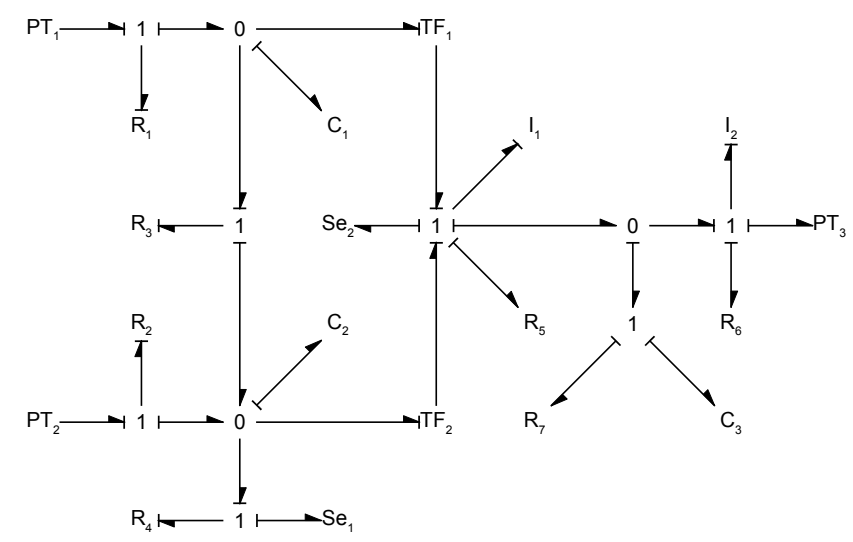

(b) Classical bond graph representation

Figure 1: Simple bond graph example

\section{BondGraph Library}

\subsection{Standard Bond Graph Elements}

The set of standard bond graph elements is comprised of basic BG elements, their modulated complements, and junctions. Thus, these are included in the BondGraph library. Therefore, the corresponding constitutive equations and element interfaces are implemented in Modelica. The power variables are made accessible by acausal connectors, whereas for signals, input-output connectors are defined compatible to blocks of the Modelica Standard Library (MSL). For the energy storage elements, initial conditions are defined locally applying start attributes for differentiated variables. The element parameters are implemented according to the BG methodology. Extending this set, sensors are modeled as simple two port elements that do not affect the power variables. Via a 
signal output interface, these elements provide power variables or their product i.e. power, corresponding time derivatives, or integrals. Again, the compatibility with blocks of the MSL is assured.

\subsubsection{Bonds}

According to the bond graph methodology, elements are interconnected by bonds. In contrast to the bonds, Modelica connections do not provide attributes for variable sign and for causality assignment, and their graphical representation. Therefore, if the bonds should be implemented closely to the original methodology, they would have to be objects of a class containing required properties. This approach would make the modeling procedure significantly more cumbersome.

The BondGraph library uses standard Modelica connections for the element interconnection introducing the connect equation directly or graphically. The graphical modeling technique is recommended here, as the BG formalism is a graphical modeling approach. Hence, an alternative method is used to determine the signs of power variables at a junction (cf. (2) and (4)). Therefore, junctions are equipped with a positive and a negative multiple port. These are indicated by a blue and a red circle, respectively. All elements connected to the positive port are considered with a positive sign $\left(s_{k}=+1\right)$ and all elements connected to the negative port are considered with a negative sign $\left(s_{k}=-1\right)$ in the sum of efforts for a 1-Junction, or in the sum of flows for a 0-Junction. In terms of the standard BG formalism, all incoming bonds are connected to the positive port and all outgoing bonds are connected to the negative port. This yields a clear graphical representation in compliance with (2) and (4) as discussed in Section 2.1.

\subsubsection{Causality Assignment}

As it has been discussed in Section 2.2, the causality assignment is not required by using Modelica. Furthermore, restrictions on algorithmic matching procedures may lead to less efficient model equations resolution. Hence, any regulations of this kind are avoided in the BondGraph library. Nevertheless, a graphical indication of the BG formalism specific causality assignment can be implemented in the animation layer extending the models of the released library by appropriate functionality. In this way, the standard BG causality assignment method might be visualized without eventual affecting of the equation resolution procedure.

\subsubsection{BondGraph Example}

The implementation of the example presented in Section 2.3 is shown in Fig. 2, now using the BondGraph library in Dymola. Here, the pressures and force imposed at the hydraulic and mechanical ports, respectively, are implemented utilizing effort sources and look-up tables from the MSL.

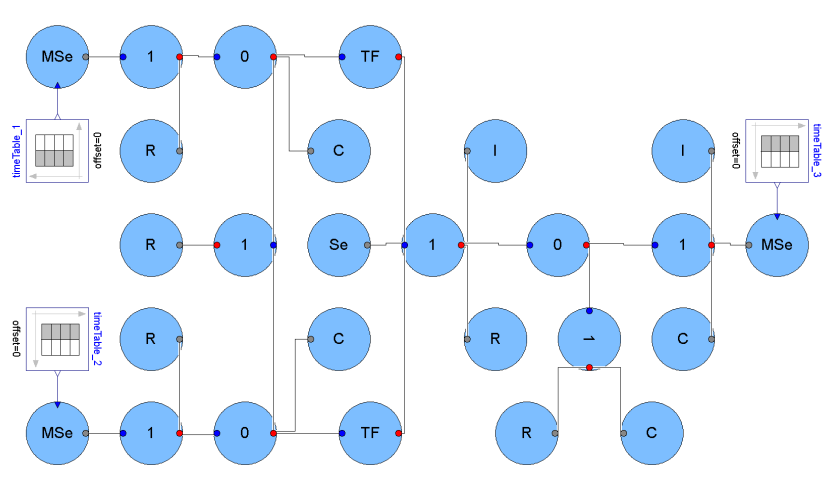

Figure 2: Simple bond graph example implemented using BondGraph

\subsection{Hydraulic Elements and Effects}

Hydraulic processes are subprocesses of fluidmechanical power exchange. For the modeled processes, this is considered transient and spatially onedimensional along a main flow direction. Models of the following hydraulic effects are included in the BondGraph library: capacitive energy storage $(H C$ and $M H C$ ), inductive energy storage $(H I)$, dissipation (HR, HRL, HRT, and MHRT), and processes with source characteristics (HSe acc and HSe ind). In the hydraulic domain, the power variables $e$ effort and $f$ flow are pressure and volume flow, respectively.

\subsubsection{Working Fluid Properties}

For the modeling of these hydraulic processes, the properties of the working fluid are of essential interest, since these may vary significantly within the working range. The hprop model of the BondGraph library provides a computation of the hydraulic fluid properties density and viscosity depending on the fluid temperature and the absolute pressure. By default, it is parameterized concerning the working fluid HLP ISO VG 32 as specified by the German industry norm DIN 54524 , part 2. The introduced model is valid for the temperature range of $[288,363] \mathrm{K}$ and the feasible pressure lies in a wide range of $\left[1,10^{8}\right] \mathrm{Pa}$. The properties computed by the hrrop model play an important role for 
other hydraulic effects and therefore it is contained in the models of hydraulic processes.

For each process, a decisive pressure is defined by the arithmetic mean of the pressures before and after the considered process. This definition assures the independence of the flow direction and is of sufficient accuracy in most cases. Different to the standard bond graph convention, hydraulic processes are implemented as two-port elements in the BondGraph library where the mean values of the pressures at the ports, and also the fluid properties as determined by hprop, are automatically considered.

The dependence of fluid properties upon other conditions besides pressure and temperature is not modeled. The working fluid is assumed to be a homogeneous mixture of hydraulic oil and air, whereby the relative mass parts in the mixture are assumed to be time and state invariant.

Density. The density of the hydraulic oil is described by the equation

$$
\rho_{\text {oil }}=\rho_{\text {oil }, \text { ref }} \cdot \exp \left(\kappa_{\text {oil }} \cdot\left(p-p_{\text {ref }}\right)-\gamma_{o i l} \cdot\left(T-T_{\text {ref }}\right)\right) \text {, }
$$

considering the compressibility and thermal expansion of the oil by the parameters $\kappa_{o i l}$ and $\gamma_{o i l}$, respectively, whereby the density of air is modeled by the ideal gas law

$$
\rho_{\text {air }}=\rho_{\text {air }, r e f} \cdot \frac{p}{p_{\text {ref }}} \cdot \frac{T_{\text {ref }}}{T} .
$$

These densities are then used to obtain the the density of the mixture by

$$
\rho=\left(\frac{\mu_{\text {oil }}}{\rho_{\text {oil }}}+\frac{\mu_{\text {air }}}{\rho_{\text {air }}}\right)^{-1},
$$

which may be parameterized by the relative mass parts of the hydraulic oil and air.

It is also possible to obtain the compensated compressibility modulus of the working fluid from this model (cf. [7]). Thus it was verified with the conversed available data for the compensated compressibility modulus.

Viscosity. The viscosity of the hydraulic oil is modeled by the Roelands relation (cf. [8])

$$
\eta_{\text {oil }}=\eta_{\text {oil }, \text { ref }} \cdot \exp \left(\ln \left(\frac{\eta_{\text {oil }, \text { ref }}}{6.315 \cdot 10^{-5} \mathrm{~Pa} \cdot \mathrm{s}}\right) \cdot \psi\right),
$$

with

$$
\psi=-1+\left(1+\frac{p-p_{r e f}}{1.96 \cdot 10^{8} \mathrm{~Pa}}\right)^{\zeta} \cdot\left(\frac{T-138 \mathrm{~K}}{T_{r e f}-138 \mathrm{~K}}\right)^{\xi} .
$$

The viscosity of air is described by the own approximation

$$
\eta_{a i r}=\eta_{\text {air } r e f} \cdot \frac{\theta(p, T)}{\theta\left(p_{r e f}, T_{r e f}\right)},
$$

with

$$
\theta(p, T)=p^{0.01} \cdot T^{0.75}+0.132 \cdot p \cdot T^{-2} .
$$

Data used for the approximation is available in [9].

For the calculation of the viscosity of the mixture, relative volume parts of the components are obtained by

$$
\phi_{o i l}=\frac{\frac{\mu_{o i l}}{\rho_{\text {oil }}}}{\frac{\mu_{\text {oil }}}{\rho_{\text {oil }}}+\frac{\mu_{\text {air }}}{\rho_{\text {air }}}}
$$

and

$$
\phi_{\text {air }}=\frac{\frac{\mu_{\text {air }}}{\rho_{\text {air }}}}{\frac{\mu_{\text {oil }}}{\rho_{\text {oil }}}+\frac{\mu_{\text {air }}}{\rho_{\text {air }}}} .
$$

With reference to it's physical characteristic, the viscosity of the homogeneous mixture of hydraulic oil and air is then described by

$$
\eta=\frac{\eta_{\text {oil }} \cdot \phi_{\text {oil }}^{2 / 3}+\eta_{\text {air }} \cdot \phi_{\text {air }}^{2 / 3}}{\phi_{\text {oil }}^{2 / 3}+\phi_{\text {air }}^{2 / 3}} .
$$

\subsubsection{Hydraulic Capacitance}

The capacitive storage capability has a significant effect on the dynamic behavior of hydraulic systems. This property results from the elasticity of the considered hydraulic component and consequently from the pressure dependent variability of the enclosed fluid volume in the component. At low pressure, the compressibility of the working fluid has a considerable effect on the capacitive storage capability. Both effects are considered by the hydraulic capacitance model $H C$ and mathematically described by

$$
f=\frac{1}{\rho} \cdot \frac{d}{d t}(\rho \cdot v)
$$

with

$$
v=v_{0} \cdot \exp \left(c_{v} \cdot\left(e-e_{0}\right)\right),
$$

where $e$ is the pressure, $e_{0}$ is the initial pressure, and $f$ is the volume flow difference taken by the component from the main volume flow.

Whereas the initial volume $v_{0}$ is a parameter in the $H C$ model, it can be adjusted by an external signal in the $M H C$ model. This extends the modeling opportunities and e.g. allows the consideration of components with moving parts as for instance cylinders. 


\subsubsection{Hydraulic Inductance}

From a power-based point of view, the inductive energy storage is the dual process to the capacitive storage and it mainly results from the inertia of the working fluid. The relation between the power variables in the inductive storage element is obtained from the principle of linear momentum applied on the enclosed working fluid in the component by

$$
e=\frac{d}{d t}\left(\frac{l \cdot \rho}{A} \cdot f\right)
$$

where $e$ is the pressure difference across the component.

\subsubsection{Hydraulic Dissipation}

Dissipative hydraulic processes are highly nonlinear and change their characteristic dependent on the transient flow conditions (cf. [10]). As the hydraulic resistance is described using different models according to the particular effect, several elements are provided by the BondGraph library: strait pipe resistance $(H R)$, resistance of a pipe fitting with laminar flow $(H R L)$, resistance of a pipe fitting with turbulent flow $(H R T)$, and switchable hydraulic resistances (MHRT) are distinguished.

Strait pipe resistance. The dissipative resistance of strait pipes is extensively studied in the literature (cf. e.g. [11], [12], [13]). The model proposed in this contribution (given by the following set of equations (19)(25)) is based on the Darcy equation (equation (19), cf. [14]). The pipe friction factor for the entire range of the Reynolds number is obtained with an explicit, continuous approximation of the pipe friction factor equation for laminar flow and of the Colebrook-White equation (cf. [15]).

As can be seen by Fig. 3, the resulting pipe friction factor function $\lambda(R e)$ (cf. equation (22)) is continuous as well as continuously differentiable, it does not diverge, and also does not include distinctions between different cases (if-then contructs), which yields stable computability. Due to the explicit form of equation (24), our approach also avoids the computationally expensive solution of the originally proposed implicit equation for turbulent flows.

$$
\begin{aligned}
& e=\lambda \cdot \frac{l \cdot \rho}{2 \cdot d_{h} \cdot A^{2}} \cdot|f| \cdot f \\
& \operatorname{Re}=\frac{d_{h} \cdot \rho \cdot|f|}{A \cdot \eta}
\end{aligned}
$$

$$
\begin{aligned}
& R e_{t}=\frac{2 \cdot 6.9}{1-\left(\frac{r_{h}}{3.7}\right)^{1.11}} \\
& \lambda=\frac{\lambda_{l}}{1+\exp \left(\frac{R e-R e_{\text {crit }}}{0.228 \cdot R e_{\text {range }}}\right)}+\frac{\lambda_{t}}{1+\exp \left(\frac{R e-R e_{\text {crit }}}{-0.228 \cdot R e_{\text {range }}}\right)}
\end{aligned}
$$

$\lambda_{l}=\frac{64}{R e+R e_{\text {min }} \cdot\left(1-\tanh \left(\frac{R e}{R e_{\text {min }}}\right)\right)}$

$\lambda_{t}=\left(\frac{-1.8}{\ln (10)} \cdot \ln \left(\left(\frac{r_{h}}{3.7}\right)^{1.11}+\chi\right)\right)^{-2}$

$$
\chi=\frac{6.9}{R e+R e_{t} \cdot\left(1-\tanh \left(\frac{R e}{R e_{t}}\right)\right)}
$$

In this equation set, $e$ is the pressure difference across the component. To obtain numerical stability using sigmoidal functions, the following parameters are utilized: $R e_{\text {crit }}$ is the Reynolds number value for the laminar-turbulent flow transition, $R e_{\text {range }}$ is the Reynolds number range for the laminar-turbulent flow transition and $R e_{\min }$ is the Reynolds number value for the zero-flow crossing.

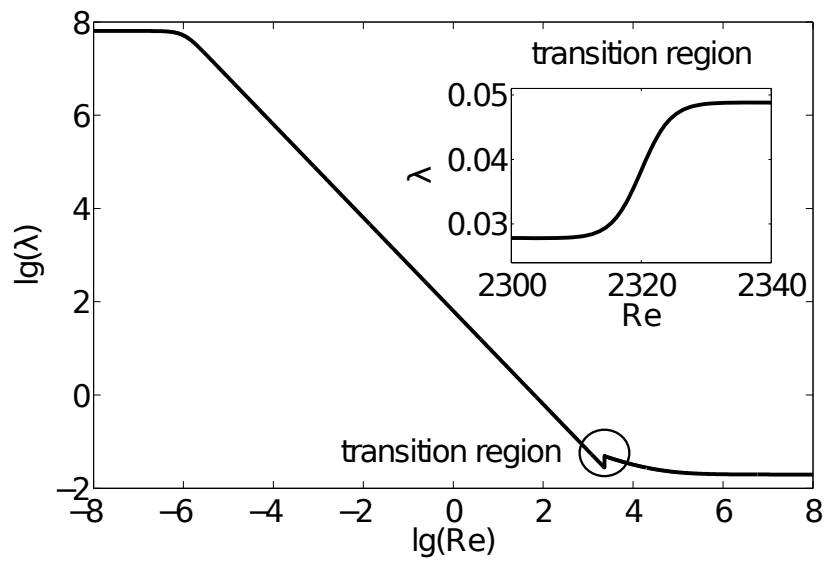

Figure 3: Proposed approximation of pipe friction factor $\lambda$ according to equation (22). The inset shows the laminar-turbulent transition region in detail with typical values for $R e_{\text {crit }}$ and $R e_{\text {range }}$.

Resistance of pipe fittings. The effect of dissipation due to hydraulic fittings is available by the models $H R L$ and $H R T$. These may be used in case of hydraulic apertures, throttles, bendings, cross-section changes, and similar components. Resistances in these components (cf. [7]) can be described by taking their flow characteristic into account. In case of laminar flow, the viscosity of the fluid strongly affects the resistance which is expressed by the equation

$$
e=\frac{e_{r e f} \cdot \eta}{\eta_{r e f} \cdot f_{r e f}^{\gamma}} \cdot|f|^{\gamma-1} \cdot f
$$


Contrariwise, in case of turbulent flow, the density of the fluid has to be considered:

$$
e=\frac{e_{r e f} \cdot \rho}{\rho_{\text {ref }} \cdot f_{r e f}^{\gamma}} \cdot|f|^{\gamma-1} \cdot f .
$$

In both cases, $e$ is the pressure drop across the component. For both laminar and turbulent resistances, often flow and pressure drop are not in linear relation to each other, but their relation may be described by a characteristic flow exponent $\gamma$, e.g. $\gamma=2$ if the flow is proportional to the square root of the effort. However, it usually depends on the geometry of the fitting and may therefore be adjusted by a parameter of the model.

Switchable hydraulic resistance. Hydraulic valves may be interpreted as switchable resistances and thus be modeled by switchable hydraulic elements. The MHRT element represents a signal controlled hydraulic resistance with turbulent characteristic where an input signal allows to control the state of the hydraulic resistance. An input value of 1 corresponds to a complete opened valve, whereas a value of 0 corresponds to a completely closed valve. To generate appropriate control inputs, several blocks are also provided by the BondGraph library. In this way the physical multi-domain nature of valves may be taken into account: though valves affect the hydraulic resistance, they are controlled by mechanical systems which are actuated either manually or by further technical units, e.g. a solenoid actuators. Therefore, array types of power-based models can be efficiently used for the modeling of hydraulic valves (cf. [16]).

\subsubsection{Processes with Source-Characteristic}

Height change. In the fluid-mechanical power exchange processes, two subprocesses with effortsource-characteristic are often involved. One of them is the pressure change $e$ due to fluid displacement in the earth gravitation field, therefore

$$
e=\rho \cdot g \cdot d
$$

which is considered by the MSe acc model.

Cross section area change. The second subprocess is covered by the MSe ind element and models the pressure change $e$ due to a cross sectional area change in hydraulic lines, thus

$$
e=\frac{\rho}{2} \cdot|f|^{2} \cdot\left(\frac{1}{A_{1}^{2}}-\frac{1}{A_{2}^{2}}\right) .
$$

\subsubsection{Consideration of Nonlinearity}

Causalized elements. As pointed out in Section 2.2, causality does not have to be assigned in general by using Modelica. Nevertheless, when using nonlinear elements e.g. HR, HRT, HRL, or MHRT, it is recommended that the designer observes whether the element has effort-out or flow-out causality in the actual model. Accordingly, the activation of an appropriate formulation of the element equations should be controlled by a parameter. In this way, unnecessary numerical inversion of the nonlinear element equations is avoidable. Hence, the solution time of the model can be shortened and the solution accuracy and stability can be increased.

For instance, in (30) and (31), both causalized forms of the turbulent resistance are given (cf. equation (27)).

$$
\begin{gathered}
e=\frac{e_{r e f} \cdot \rho}{\rho_{r e f} \cdot f_{r e f}^{\gamma}} \cdot|f|^{\gamma-1} \cdot f \\
f=\left(\frac{e_{r e f} \cdot \rho}{\rho_{r e f} \cdot f_{r e f}^{\gamma}}\right)^{\frac{-1}{\gamma}} \cdot|e|^{\frac{1}{\gamma}-1} \cdot e
\end{gathered}
$$

In (32), a form corresponding to the linear resistance element is obtained. The state dependent resistance coefficient $r$ is separated. Different formulations for the resistance coefficient are then used dependent on the par $_{\text {caus }}$ parameter.

$$
e=r \cdot f
$$

with

$$
r= \begin{cases}\frac{e_{\text {ref }} \cdot \rho}{\rho_{\text {ref }} \cdot f_{\text {ref }}^{\gamma}} \cdot|f|^{\gamma-1} \cdot f & \text { if } \text { par }_{\text {caus }}=1, \\ \left(\frac{e_{\text {ref }} \cdot \rho}{\rho_{\text {ref }} \cdot f_{\text {ref }}^{\gamma}}\right)^{\frac{1}{\gamma}} \cdot|e|^{1-\frac{1}{\gamma}} \cdot e & \text { if } \text { par }_{\text {caus }}=2\end{cases}
$$

Variation of the proportionality factors. Corresponding reformulations and separations of the proportionality factors are often possible and convenient for nonlinear elements with other characteristics. As these coefficients in such reformulated relations are in general time and state variant, their absolute values can attain low values or zero. The same has to be considered for the reciprocal of the absolute value. In these cases, structural changes arise in the interconnected model equations and a numerical solution is either not possible or becomes unstable. For instance, this might result in a non-decaying oscillation of the power variables where the amplitude and particularly the frequency of the oscillation depend on the chosen 
solver algorithm and solution tolerance. In this case, an effective and simple modification of the model is the limitation of the value of the proportionality coefficient. The mentioned numerically induced oscillations are avoidable completely by this model extension.

\subsection{Comparison Remark to BondLib}

BondLib is a former successful implementation attempt of the bond graph formalism in the Modelica language presented in [17] awarded as the best free Modelica library in the framework of the Modelica Conference 2005. BondGraph is a conceptually different modeling library, intended rather for pragmatic practical modeling using bond graph methodology then for educational purpose. In contrast to BondLib, BondGraph does not include elements closely corresponding to bonds. Instead, Modelica standard connections are utilized for the interconnection of BG elements. Consequently, the assignment of the variable signs is implemented as a property of junctions instead of using the directional property of bonds. In this way, BondGraph utilizes Modelica more efficiently slightly differing from the graphical representation defined in the BG methodology. Since bonds as elements are excluded, in comparison to a model set up with BondLib, an equivalent model set up with BondGraph involves significantly less elements. Furthermore, the graphical clarity for large models can be maintained more straightforward. The junction implementation in BondGraph permits connection of elements of unlimited number, whereas in the BondLib, junctions with different fixed numbers of ports are available. Hence, model modifications and expansions are performed more efficiently utilizing BondGraph, since originally used junctions may be retained. From this point of view, the BondGraph junction implementation is closer to the BG methodology. The BondLib library offers bonds with fixed causality. As described before, BondGraph avoids any regulations of this kind well motivated following more closely the Modelica approach. Concluding this short comparison, we suggest BondGraph for practical modeling, whereas we recognize that BondLib offers several properties very important for bond graph beginners and educational purpose.

\section{Industrial Hydraulic Plant}

The discussed library is utilized for the modeling of an industrial hydraulic plant. The considered plant is a riveting system with a hydraulic power transmission. The system can be structured into the following principal units: a hydraulic power supply unit containing an inductance motor and a pump, a valve block, a hydraulic-mechanical actuator, hydraulic pipes interconnecting these units, and a sequence control. Hence, the model to be developed should cover a comprehensive hydraulic network. Figure 4 shows the model top layer.

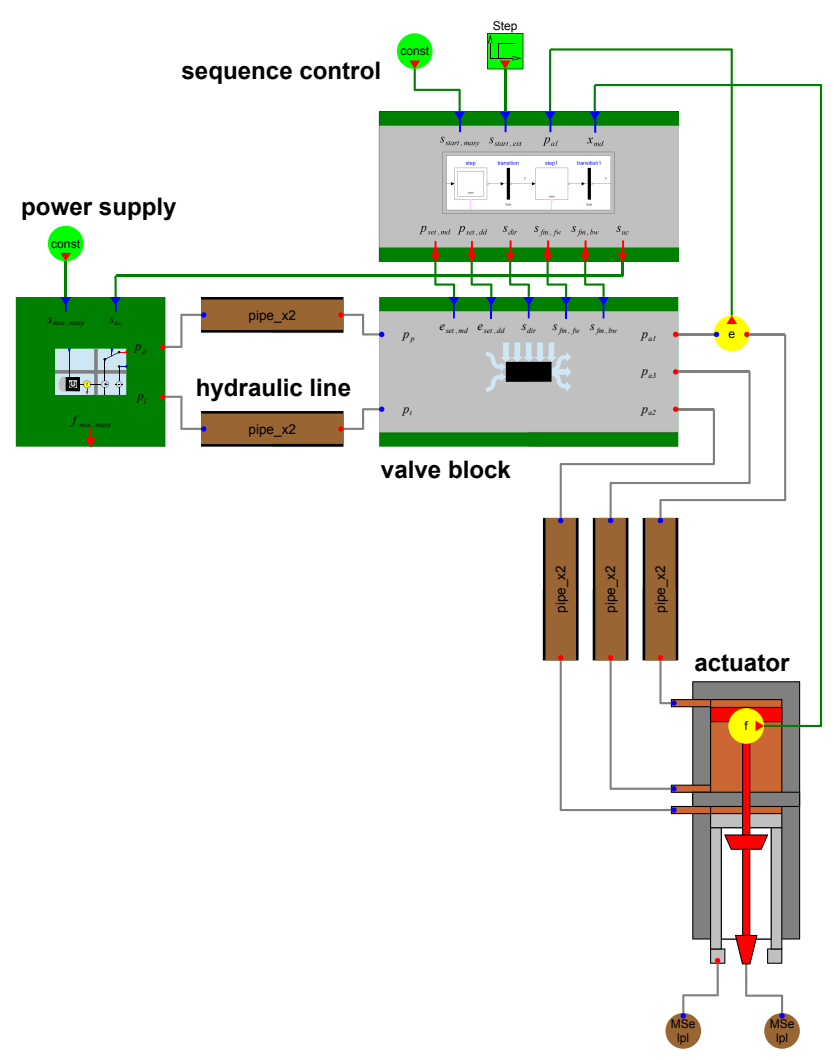

Figure 4: Model of the considered riveting system

The submodels representing principal units are modeled using the BondGraph library and the MSL. A plain example for this model set-up is the model of a hydraulic pipe consisting of hydraulic elements exclusively. A series of hydraulic dissipative and inductive elements separated by hydraulic capacitive elements may be utilized for the description of the pipe behavior. The series starts and ends with turbulent resistances representing resistances of the fittings. The inner part is the repeating of series consisting of a straight pipe resistance and a hydraulic inductance again separated by capacitive elements. This repeating incorporates spatial discretization of the pipe along the main flow direction. Consequently it should be undertaken an appropriate number of times to cover relevant system eigenvalues by the model. An example of pipe 


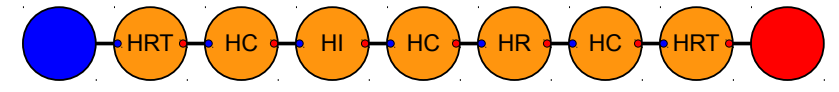

Figure 5: Model of the hydraulic pipe

model with one inductive storage is given in Fig. 5.

The model of the complete plant is a higher index differential-algebraic system consisting of 4923 equation with 106 continuous time states. The operation cycle of the plant of $1.7 \mathrm{~s}$ is simulated. Figure 6 shows the normalized displacement $x$ of the actuator effector, the mechanical energy $E$ supplied by the inductance motor in the power supply unit, and the pressure $p$ in the hydraulic circuit.

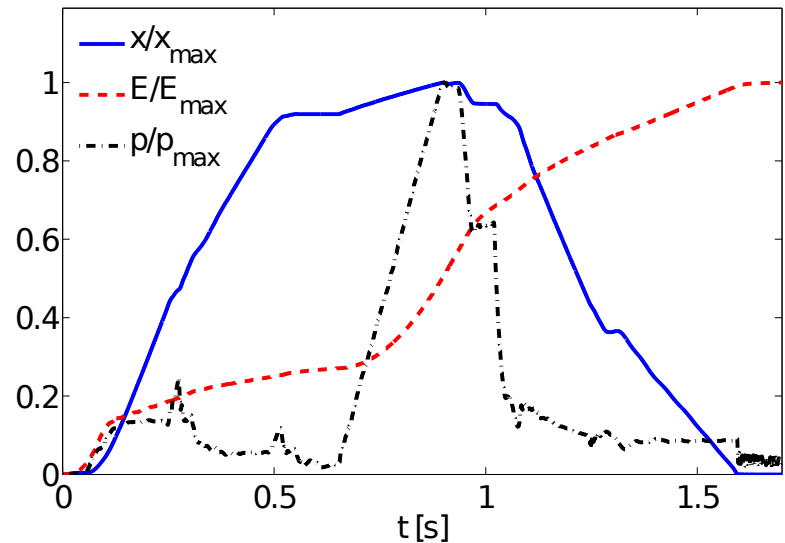

Figure 6: Simulation results. Plotted are the displacement $x$ of the actuator, the mechanical energy $E$ of the inductance motor, and the pressure $p$ supplied by the pump.

The model parameters are identified by an optimization approach with reference to measurements obtained at a demonstrator plant. Hence, the model is verified against comprehensive experimental data. Furthermore, simulation based optimization of the plant is succeeded with the objective function defined as the consumed energy for an operation cycle (cf. [18]).

\section{Conclusion}

The bond graph methodology is a generalized powerbased modeling approach. Hence, it is particularly advantageous for modeling of multidisciplinary systems. Therefore, BondGraph, the Modelica implementation of this approach enables applicants to take advantage of both, the flexible Modelica language and the well structured generalized power-based bond graph formalism. Furthermore, utilizing Modelica as the im- plementation language, efficient general purpose modeling and simulation environments are made available for the bond graph applicants. Besides the development of BondGraph, a practical application of the library is conducted. Thereby, an industrial hydraulic plant is modeled, simulated, and optimized utilizing the developed modeling library.

\section{Acknowledgments}

This elaboration was co-funded by the European Union (European Funds for Regional Development) and North Rhine-Westphalia, Germany (Federal State of Germany).

\section{References}

[1] D. Jeltsema and J. M. A. Scherpen, "Multidomain modeling of nonlinear networks and systems," IEEE Control Systems Magazine, vol. 29, pp. 28-59, 2009.

[2] R. A. Layton, Principles of Analytical System Dynamics. Springer, 1998.

[3] W. Borutzky, Bond Graph Methodology. Springer, 2010.

[4] I. Alkov and R. Diekmann. (2013) BondGraph library. [Online]. Available: https://www.modelica.org/libraries

[5] H. M. Paynter, Analysis and Design of Engineering Systems. M.I.T. Press, 1961.

[6] J. F. Broenink, "20-sim software for hierarchical bond-graph/block-diagram models," Simulation Practice and Theory, vol. 7, no. 5-6, pp. 481492, 1999.

[7] H. Murrenhoff, Grundlagen der Fluidtechnik Teil 1: Hydraulik. Shaker, 2012.

[8] C. J. A. Roelands, J. C. Vlugter, and H. I. Waterman, "The viscosity-temperature-pressure relationship of lubricating oils and its correlation with chemical constitution," ASME Journal of Basic Engineering, vol. 11, pp. 601-611, 1963.

[9] J. H. Spurk and N. Aksel, Strömungslehre, Einführung in die Theorie der Strömungen. Springer, 2010. 
[10] R. Etlender, "Modellierung und Simulation der Wellenausbreitung in flexiblen hydraulischen Leitungen," Ph.D. dissertation, Universität Stuttgart, 2012.

[11] J. Nikuradse, "Strömungsgesetze in rauhen Rohren," V.D.I. Forschungsheft, vol. 361, pp. 122, 1933.

[12] C. F. Colebrook, "Turbulent flow in pipes, with particular reference to the transition region between the smooth and rough pipe laws," Journal of Institution of Civil Engineers, vol. 11, no. 4, pp. 133-156, 1939.

[13] L. F. Moody, "Friction factors for pipe flow," Transactions of the ASME, vol. 66, pp. 671-684, 1944.

[14] H. Darcy, Les Fontaines Publiques de la Ville de Dijon. Dalmont, 1856.

[15] S. E. Haaland, "Simple and explicit formulas for the friction factor in turbulent pipe flow," Transactions of the ASME, Journal of Fluids Engineering, vol. 105, pp. 89-90, 1983.

[16] I. Alkov, "Verallgemeinertes Modell hydraulischer Ventile," 2012, FLUIDON Conference 2012.

[17] F. E. Cellier and A. Nebot, "The Modelica bond graph library," Proceedings of the 4th International Modelica Conference, pp. 57-65, 2005.

[18] J. Heinze, R. Diekmann, I. Alkov, and D. Weidemann, "Model-based energy optimization of assembly systems," 2013, 35th IAT Colloquium of Automation.

\section{Appendix}

\section{List of Formula Symbols}

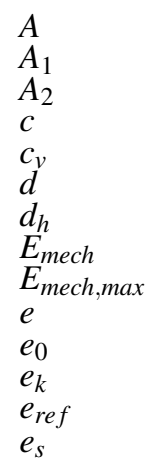

cross section area of enclosed fluid volume cross section area before change cross section area behind change capacitance coefficient

volumetric compliance factor

height difference

hydraulic diameter

mechanical energy

maximal mechanical energy value

effort

initial effort

effort at port $k$

reference effort

$e_{s}$

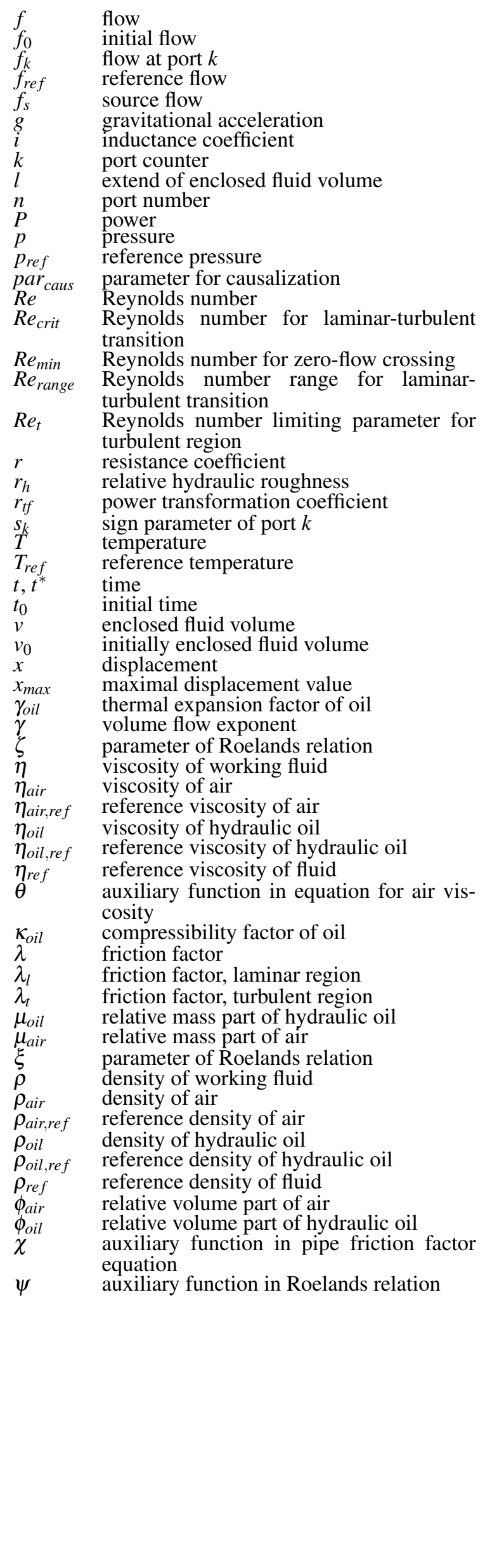

